Related content

Fluid description of Weibel-type instabilities via full pressure tensor dynamics

To cite this article: M. Sarrat et al 2016 EPL 11545001

$$
\begin{aligned}
& \text { - Multi-stream Vlasov model for the study of } \\
& \frac{\text { relativistic Weibel-type instabilities }}{\text { A Inglebert, A Ghizzo, T Reveille et al. }} \\
& \text { - 'Magneto-elastic' waves in an anisotropic } \\
& \frac{\text { magnetised plasma }}{\text { D Del Sarto, F Pegoraro and A Tenerani }} \\
& \text { - A multi-stream Vlasov modeling unifying } \\
& \frac{\text { relativistic Weibel-type instabilities }}{\text { A. Inglebert, A. Ghizzo, T. Reveille et al. }}
\end{aligned}
$$

View the article online for updates and enhancements. 


\title{
Fluid description of Weibel-type instabilities via full pressure tensor dynamics
}

\author{
M. Sarrat, D. Del SArto ${ }^{(a)}$ and A. Ghizzo \\ Institut Jean Lamour, UMR 7198 CNRS - Université de Lorraine - Vandœuvre-lès-Nancy, France
}

received 1 July 2016; accepted in final form 24 August 2016

published online 14 September 2016
PACS 52.35.Qz - Microinstabilities (ion-acoustic, two-stream, loss-cone, beam-plasma, drift, ion- or electron-cyclotron, etc.)
PACS 52.25.Dg - Plasma kinetic equations
PACS 52.65.Kj - Magnetohydrodynamic and fluid equation

\begin{abstract}
We discuss a fluid model for the description of Weibel-type instabilties based on the inclusion of the full pressure tensor dynamics. The linear analysis first performed by BASU B., Phys. Plasmas, 9, (2002) 5131, for the strong anisotropy limit of Weibel's instability is extended to include the coupling between pure Weibel's and current filamentation instability, and the potential of this fluid approach is further developed. It is shown to allow an easier interpretation of some physical features of these coupled modes, notably the role played by thermal effects. It can be used to identify the role of different closure conditions in pressure-driven instabilities which can be numerically investigated at a remarkably lower computational cost than with kinetic simulations.
\end{abstract}

Copyright (C) EPLA, 2016

Introduction. - In this letter we discuss a model for anisotropy-driven instabilities of Weibel type $[1,2]$, based on a fluid description in which a full pressure tensor evolution is retained. This approach makes it possible to identify and understand from a macroscopic point of view some of their kinetic features, whose physical interpretation appears difficult when a full kinetic model is adopted. Besides, it opens to the investigation of the role of different closure conditions on the linear and nonlinear dynamics of Weibel-type modes, and, from a computational point of view, it represents an important saving factor in comparison to a full kinetic approach.

The possibility of such an extended fluid description was first evidenced by Basu [3], who showed how to recover, in the model we consider here, the strong anisotropy limit of the classical dispersion relation, obtained by Weibel [1] for the growth rate of a quasi-static magnetic field generated by the phase-space anisotropy of a bi-Maxwellian electron distribution function in a neutralizing bulk ion background (pure "Weibel instability", or WI hereafter). While providing a physical interpretation of the latter, on the other hand, Fried himself implicitly suggested the possibility to give a fluid picture of these instabilities: introducing what would have been later named counterstreaming "current filamentation

\footnotetext{
(a) E-mail: daniele.del-sarto@univ-lorraine.fr
}

instability" (CFI) or [4] "streaming Weibel" instability, he showed that the temperature anisotropy in Weibel's analysis could be substituted by the momentum anisotropy of counterstreaming electron beams, represented by two Dirac's $\delta$-functions differently centred in the velocity space [2]. Since then, cold fluid models have been used to describe both the linear stage [5] of the relativistic and non-relativistic CFI, possibly in the presence of spatial resonances effects [6,7], and some features of its nonlinear dynamics, such as the saturation at "kinetic" scales [8] (i.e., the electron skin depth $d_{e} \equiv c / \omega_{p e}$ ), the onset of secondary magnetic reconnection processes [9], or the coupling with the two-stream instability (TSI) in a 3D, spatially inhomogeneous configuration [10]. However, some of the conclusions drawn within these cold models, such as the complete de-coupling between the CFI purely electromagnetic (e.m.) and electrostatic features [10] in some limits, have proven not to survive in a full kinetic treatment with non-null beam temperatures [11-13]. Kinetic features have been instead successfully described by means of kinetic reduced models based on specific classes of electron distribution functions, such as waterbags [14-17], multi-stream distributions [18], combinations of the two [19] or some of their special cases, such as "multi-ring" distributions [20-22]. While on the one hand such special classes of particle distributions 
are useful both for the numerical initialisation of these instabilities, especially in the relativistic regime, and for their numerical simulation at a lower computational cost, on the other hand the exact analytical calculations they typically allow provide a valuable insight into the physics of Weibel-type modes. The multi-stream description, for example, has provided a unifying framework for the description of both pure WI and CFI, by highlighting the representability of a temperature anisotropy as a momentum anisotropy $[18,19]$.

The aim of this letter is to discuss the analogous usefulness of the extended fluid model we consider here, somehow a "reduced kinetic model", since the inclusion of the full pressure tensor dynamics allows a consistent description of quadrupole momentum deformations of the distribution function, as evidenced in ref. [23] for the case of anisotropic ions and cold massless electrons. With this model, for example, it has been shown [24] how a sheared velocity field provides a dynamical mechanism for the generation of non-gyrotropic pressure anisotropy via the transfer of space inhomogeneities of Vlasov's 1storder moment to an anisotropic space inhomogeneity of the pressure tensor components. Here, by considering two anisotropic, non-relativistic electron fluids, coupled to a neutralising hydrogen ion background by Maxwell's equations, we extend Basu's preliminar analysis [3] to discuss the linear coupling between WI and CFI. In this way we identify some features whose interpretation was not yet provided, in such simple terms, within a fully kinetic description.

Physical model. - We consider two non-relativistic, counterpropagating electron beams, by allowing each beam's pressure tensor to be anisotopic in its own rest frame, i.e., by allowing for different values of the pressure (or "temperature") along the principal axes of the second-order moment tensor of each electron population - see fig. 1. The fluid model we adopt can be extended to include anisotropic ions but we restrict here to time intervals $\Delta t \lesssim \omega_{p i}^{-1}$, with $\omega_{p i}=c / d_{i}$ ion plasma frequency. Ions will therefore provide a static, neutralising background with uniform density $n_{i}$, constant in time. We write the first three Vlasov' moments equations, as derived and discussed, e.g., in the appendix of [23] (note that $\alpha$ labels particle species, there, instead) for two electron fluids labelled with the index $\alpha=1,2$ and for an electron mass $m$ :

$$
\begin{aligned}
& \partial_{t} n_{\alpha}+\nabla \cdot\left(\boldsymbol{u}_{\alpha} n_{\alpha}\right)=0 \\
& \partial_{t} \boldsymbol{u}_{\alpha}+\boldsymbol{u}_{\alpha} \cdot \boldsymbol{\nabla} \boldsymbol{u}_{\alpha}=-\frac{e}{m c}\left(c \boldsymbol{E}+\boldsymbol{u}_{\alpha} \times \boldsymbol{B}\right)-\frac{\boldsymbol{\nabla} \cdot \boldsymbol{\Pi}_{\alpha}}{n_{\alpha} m}, \\
& \partial_{t} \boldsymbol{\Pi}_{\alpha}+\boldsymbol{\nabla} \cdot\left(\boldsymbol{u}_{\alpha} \boldsymbol{\Pi}_{\alpha}\right)+\boldsymbol{\nabla} \boldsymbol{u}_{\alpha} \cdot \boldsymbol{\Pi}_{\alpha}+\left(\boldsymbol{\nabla} \boldsymbol{u}_{\alpha} \cdot \boldsymbol{\Pi}_{\alpha}\right)^{T}= \\
& -\frac{e}{m c}\left(\boldsymbol{\Pi}_{\alpha} \times \boldsymbol{B}+\left(\boldsymbol{\Pi}_{\alpha} \times \boldsymbol{B}\right)^{T}\right)-\boldsymbol{\nabla} \cdot \boldsymbol{Q}_{\alpha} .
\end{aligned}
$$

Apex " $T$ " expresses matrix transpose, $\boldsymbol{\Pi}_{\alpha} \equiv n_{\alpha} m\left(\langle\boldsymbol{v} \boldsymbol{v}\rangle_{\alpha}-\right.$ $\left.\boldsymbol{u}_{\alpha} \boldsymbol{u}_{\alpha}\right)$ is the pressure tensor and $\boldsymbol{Q}_{\alpha} \equiv m n_{\alpha}\langle(\boldsymbol{v}-$ $\left.\left.\boldsymbol{u}_{\alpha}\right)\left(\boldsymbol{v}-\boldsymbol{u}_{\alpha}\right)\left(\boldsymbol{v}-\boldsymbol{u}_{\alpha}\right)\right\rangle_{\alpha}$ is the heat flux tensor, where $\langle\ldots\rangle_{\alpha}$ indicates average in the velocity coordinates $\boldsymbol{v}$ with respect

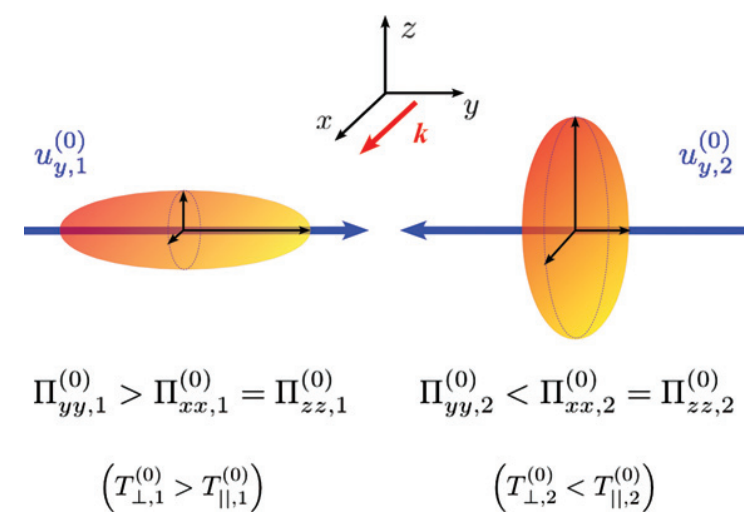

Fig. 1: (Colour online) Example of asymmetric beam configuration: two counterpropagating beams with initial pressure anisotropy, in each own reference frame, between the components parallel ("|") and perpendicular (" $\perp$ ") to the wave vector direction $\left(\boldsymbol{e}_{x}\right)$. In the limit of vanishing initial velocities, $u_{y, 1}^{(0)}=u_{y, 2}^{(0)}=0$, the population (1) would be intrinsically unstable to a pure WI whereas the population (2) would be not.

to the particle distribution $f_{\alpha}(\boldsymbol{x}, \boldsymbol{v})$. Where specified, for an isotropic pressure $\boldsymbol{\Pi}_{\alpha}=P_{\alpha} \mathbf{I}$, eq. (3) will be replaced by an indipendent closure condition, generally unrelated to eq. (3), with polytropic index $\Gamma_{\alpha}$,

$$
\partial_{t} P_{\alpha}+\boldsymbol{u}_{\alpha} \cdot \nabla P_{\alpha}+\Gamma_{\alpha} P_{\alpha} \nabla \cdot \boldsymbol{u}_{\alpha}=0 .
$$

While at equilibrium (apex "0") we assume $\boldsymbol{J}^{(0)}=$ $-e \sum_{\alpha} n_{\alpha}^{(0)} \boldsymbol{u}_{\alpha}^{(0)}=0$ and quasi-neutrality, $\sum_{\alpha} n_{\alpha}^{(0)}=n_{i}^{0}$, with $\boldsymbol{J} \equiv-e \sum_{\alpha} n_{\alpha} \boldsymbol{u}_{\alpha}$, in general we consider eqs. (1)-(4) coupled to the full set of Maxwell's equations,

$$
\begin{array}{ll}
\partial_{t} \boldsymbol{B}=-c \boldsymbol{\nabla} \times \boldsymbol{E}, & \boldsymbol{\nabla} \cdot \boldsymbol{B}=0, \\
\partial_{t} \boldsymbol{E}=c \boldsymbol{\nabla} \times \boldsymbol{B}-4 \pi \boldsymbol{J}, & \boldsymbol{\nabla} \cdot \boldsymbol{E}=4 \pi e\left(n_{i}^{0}-\sum_{\alpha} n_{\alpha}\right) .
\end{array}
$$

At equilibrium we assume the beams along the $y$-axis, $\boldsymbol{J}^{(0)}=J_{y}^{(0)} \boldsymbol{e}_{y}$ and, in the most general case, a diagonal pressure tensor with non-null components of values $\Pi_{x x}^{(0)}, \Pi_{y y}^{(0)}, \Pi_{z z}^{(0)}$, uniform in space but in principle different one from each other. Such anisotropic configuration is assumed as a simplified initial condition for the onset of "pure Weibel" modes [1]. It has been shown [24], however, that sufficiently long-lived pressure anisotropies can be generated by an initial velocity shear, and, in ref. [25], anisotropic equilibrium solutions in the presence of spatial inhomogeneities have been evaluated. Spatial inhomogeneities in the initial equilibrium profile are known to affect the linear and nonlinear evolution of Weibel modes via spatial resonances $[6,7,10]$, but such an occurrence in this model is beyond the purpose of the present letter. For further simplification and since we aim at focussing on the role played by temperature effects in the coupling between transverse and longitudinal features, we consider only orthogonal perturbations with $\boldsymbol{k}=k_{x} \boldsymbol{e}_{x}$. Due to the cylindrical symmetry of the equilibrium around the 
$y$-axis for $\Pi_{x x}^{(0)}=\Pi_{z z}^{(0)}$, the solutions for $\boldsymbol{k}=k_{z} \boldsymbol{e}_{z}$ could be easily deduced from the ones we consider here after permutation of the axes and substitution of the relevant equilibrium pressure components. It is useful to introduce the squared velocities

$$
c_{x, \alpha}^{2} \equiv \frac{\Pi_{x x, \alpha}^{(0)}}{m n_{\alpha}^{(0)}}, \quad c_{y, \alpha}^{2} \equiv \frac{\Pi_{y y, \alpha}^{(0)}}{m n_{\alpha}^{(0)}}, \quad c_{z, \alpha}^{2} \equiv \frac{\Pi_{z z, \alpha}^{(0)}}{m n_{\alpha}^{(0)}},
$$

related to the respective temperatures by $k_{B} T_{x, \alpha} \equiv$ $\Pi_{x x, \alpha}^{(0)} / n_{\alpha}^{(0)}, k_{B} T_{y, \alpha} \equiv \Pi_{y y, \alpha}^{(0)} / n_{\alpha}^{(0)}, k_{B} T_{z, \alpha} \equiv \Pi_{z z, \alpha}^{(0)} / n_{\alpha}^{(0)}$, and the squared plasma frequency of each initial beam $\alpha$ and of their sum,

$$
\omega_{p e, \alpha}^{2} \equiv \frac{4 \pi n_{\alpha}^{(0)} e^{2}}{m}, \quad \omega_{p e}^{2} \equiv \omega_{p e, 1}^{2}+\omega_{p e, 2}^{2} .
$$

Perturbed quantities have the superscript " ". Unless otherwise specified (cf. the comments on the pure WI and the discussion at the end of the letter) we will completely neglect in eq. (3) the contribution of the heat flux, whose components evolve according to the equation

$$
\begin{aligned}
& \partial_{t} \boldsymbol{Q}_{\alpha}+\boldsymbol{\nabla} \cdot\left(\boldsymbol{u}_{\alpha} \boldsymbol{Q}_{\alpha}+\boldsymbol{R}_{\alpha}\right)+\left[\boldsymbol{Q}_{\alpha} \cdot \boldsymbol{\nabla} \boldsymbol{u}_{\alpha}\right]_{s y m}= \\
& {\left[\frac{\boldsymbol{\Pi}_{\alpha}}{n_{\alpha} m} \cdot \boldsymbol{\nabla} \boldsymbol{\Pi}_{\alpha}\right]_{s y m}+\frac{e}{m c}\left[\boldsymbol{Q}_{\alpha} \times \boldsymbol{B}\right]_{s y m},}
\end{aligned}
$$

where [ ] sym expresses symmetrisation with respect to the tensor components (e.g., $[\boldsymbol{u} \boldsymbol{\Pi}]_{\text {sym }}$ shall be read $u_{i} \Pi_{j k}+$ $u_{j} \Pi_{k i}+u_{k} \Pi_{i j}$ in tensorial notation $)$ and $\boldsymbol{R}_{\alpha} \equiv m n_{\alpha}\langle(\boldsymbol{v}-$ $\left.\left.\boldsymbol{u}_{\alpha}\right)\left(\boldsymbol{v}-\boldsymbol{u}_{\alpha}\right)\left(\boldsymbol{v}-\boldsymbol{u}_{\alpha}\right)\left(\boldsymbol{v}-\boldsymbol{u}_{\alpha}\right)\right\rangle_{\alpha}$ is the fourth-order moment.

Coupled WI-CFI modes. - Linear perturbations of eqs. (1)-(3), (5), (6) on the initial equilibrium above allow the excitation of both WI and CFI, coupled or separately. Before to consider in detail the latter case, we discuss the linear analysis of the coupled WI-CFI mode, considered, e.g., in ref. [13] in a kinetic regime, though mainly coupled to the TSI or in the presence of relativistic effects. The dispersion matrix $D_{i j}$, defined by $[\boldsymbol{D}] \cdot \boldsymbol{E} \equiv 0$, reads

$$
[\boldsymbol{D}]=\left(\begin{array}{ccc}
D_{x x} & D_{x y} & 0 \\
D_{x y} & D_{y y} & 0 \\
0 & 0 & D_{z z}
\end{array}\right)
$$

with

$$
\begin{aligned}
D_{x x}= & \frac{\omega^{2}}{c^{2}}-\frac{\omega^{2}}{c^{2}} \sum_{\alpha} \frac{\omega_{p e, \alpha}^{2}}{\omega^{2}-3 k_{x}^{2} c_{x, \alpha}^{2}}, \\
D_{x y}= & -\frac{\omega^{2}}{c^{2}} \sum_{\alpha} \frac{k_{x} u_{y, \alpha}^{(0)}}{\omega}\left(\frac{\omega_{p e, \alpha}^{2}}{\omega^{2}-3 k_{x}^{2} c_{x, \alpha}^{2}}\right), \\
D_{y y}= & \frac{\omega^{2}}{c^{2}}-k_{x}^{2}-\sum_{\alpha} \frac{\omega_{p e, \alpha}^{2}}{c^{2}}\left(\frac{\omega^{2}+k_{x}^{2}\left(c_{y, \alpha}^{2}-c_{x, \alpha}^{2}\right)}{\omega^{2}-k_{x}^{2} c_{x, \alpha}^{2}}\right. \\
& \left.+\frac{\left(k_{x} u_{y, \alpha}^{(0)}\right)^{2}}{\omega^{2}-3 k_{x}^{2} c_{x, \alpha}^{2}}\right), \\
D_{z z}= & \frac{\omega^{2}}{c^{2}}-k_{x}^{2}-\sum_{\alpha} \frac{\omega_{p e, \alpha}^{2}}{c^{2}}\left(\frac{\omega^{2}+k_{x}^{2}\left(c_{z, \alpha}^{2}-c_{x, \alpha}^{2}\right)}{\omega^{2}-k_{x}^{2} c_{x, \alpha}^{2}}\right) .
\end{aligned}
$$

The pure WI and cold CFI dispersion relations, which we will separetely discuss in the following sections, are recovered from the condition $\operatorname{det}[\boldsymbol{D}]=0$, respectively for $\alpha=1$ (just one "beam" with $\boldsymbol{u}^{(0)}=0$ ) and for $c_{i, \alpha}=0$ with $i=x, y, z$ (cold beams). The roots of $D_{z z}=0$ are decoupled from the others since related to a "pure" WI contribution that generates a $\tilde{B}_{y}$ component, which does not affect the dynamics of the beams to which it is colinear. The coupled WI-CFI mode is described instead by the roots of $D_{x x} D_{y y}-D_{x y}^{2}=0$. The $D_{x x}$ contribution is related to Bohm-Gross oscillations, inherently electrostatic in nature, which, for $D_{x y} \neq 0$, couple to the transverse e.m. modes related to $D_{y y}$. As already noted by Bret et al. [12] in a kinetic framework, the angle $\vartheta$ between the wave vector and the electric field, defined by $\boldsymbol{\nabla} \cdot \boldsymbol{E}=k E \cos (\vartheta)$, satisfies

$$
\tan (\vartheta) \equiv \frac{\tilde{E}_{y}}{\tilde{E}_{x}}=\frac{\left|D_{x x}\right|}{\left|D_{x y}\right|} .
$$

For $\boldsymbol{k} \cdot \boldsymbol{u}^{(0)}=0$ it identifies a purely transverse, e.m. perturbation when $\vartheta=\pi / 2$, that is, if $D_{x y}=0$. The fluid analysis we here present allows a simple physical interpretation of this requirement: $D_{x y}=0$ generalises the condition of initial current density equilibrium for the CFI, i.e., of null, global charge density flux, to the condition of null rate compressional work. The condition $\sum_{\alpha} u_{y, \alpha}^{(0)} \omega_{p e, \alpha}^{2}=$ 0 is imposed because of $u_{y, \alpha}^{(0)} \omega_{p e, \alpha}^{2}=-4 \pi e J_{y, \alpha}^{(0)} / m$ and $\sum_{\alpha} \boldsymbol{J}_{\alpha}^{(0)}=0$, and therefore $D_{x y}=0$ only if $c_{x, 1}^{2}=c_{x, 2}^{2}$. Using eqs. (7), the latter statement means $\Pi_{x x, 1}^{(0)} / n_{1}^{(0)}=$ $\Pi_{x x, 2}^{(0)} / n_{2}^{(0)}$, which for initially Maxwellian distribution functions means equal beams temperatures, as first noticed in refs. [11,12] in a Vlasov description and in ref. [20] in a multi-stream model. Using again the initial zerocurrent condition, the equivalence of the two thermal velocities corresponds to

$$
\Pi_{x x, 1}^{(0)} u_{y, 1}^{(0)}+\Pi_{x x, 2}^{(0)} u_{y, 2}^{(0)}=0 .
$$

In this case where just perturbations transverse to the beams are excited, the decoupling between electrostatic and e.m. modes is therefore granted if at equilibrium there is no neat transport, due to the beams, of the pressure tensor component across the surface orthogonal to the wave vector, that is, if the rates of compressional work of each beam per unit area through the surface orthogonal to the perturbation balance each other.

Let us now consider the special case of perfectly symmetric beams with $u_{y, 1}^{(0)}=-u_{y, 2}^{(0)}=u_{0}, n_{\alpha}^{(0)}=n_{i}^{(0)} / 2$, $c_{x, \alpha}=c_{x}, c_{y, \alpha}=c_{y} \forall \alpha$, and $c_{x} \neq c_{y}$. In this case a purely e.m. WI-CFI coupled mode exists, which solves $D_{y y}=0$. The latter can be written as

$$
\begin{aligned}
& \omega^{6}-\omega^{4}\left[\omega_{p e}^{2}+k_{x}^{2}\left(c^{2}+c_{x}^{2}\right)+3 k_{x}^{2} c_{x}^{2}\right] \\
& +\omega^{2}\left[3 k_{x}^{4} c_{x}^{4}+4 k_{x}^{4} c^{2} c_{x}^{2}+\omega_{p e}^{2} k_{x}^{2}\left(4 c_{x}^{2}-u_{0}^{2}-c_{y}^{2}\right)\right] \\
& +\omega_{p e}^{2} k_{x}^{4}\left[u_{0}^{2} c_{x}^{2}+3 c_{x}^{2}\left(c_{y}^{2}-c_{x}^{2}\right)\right]-3 k_{x}^{6} c^{2} c_{x}^{4}=0
\end{aligned}
$$


This coupled mode has a cut-off wave number satisfying $\omega\left(k_{c}\right)=0$, given by

$$
k_{c}=\frac{\omega_{p e}}{c} \sqrt{\frac{c_{y}^{2}-c_{x}^{2}}{c_{x}^{2}}+\frac{u_{0}^{2}}{3 c_{x}^{2}}},
$$

which consists in the Pythagorean addition of the cut-off wave numbers of the "fluid" WI and CFI, respectively,

$$
k_{c}^{W I} d_{e}=\sqrt{\frac{c_{y}^{2}-c_{x}^{2}}{c_{x}^{2}}}=\sqrt{\frac{T_{y}}{T_{x}}-1}, \quad k_{c}^{C F I} d_{e}=\frac{\left|u_{0}\right|}{\sqrt{3} c_{x}} .
$$

This symmetry between the WI and CFI contribution is mirrored by the approximated roots of eq. (17) obtained in the strong anisotropy, non-relativistic limit, $c_{x}^{2} \ll c_{y}^{2} \ll c^{2}$, combined with the assumption $k_{x}^{2} c_{x}^{2} / \omega^{2} \ll 1$, well verified for both WI and CFI separately as long as $k<$ $k_{c}$ (fig. 2). This root corresponds to a growing, nonpropagating mode, with growth rate

$$
\gamma_{W I / C F I} \simeq \omega_{p e} \sqrt{\frac{k_{x}^{2}\left(c_{y}^{2}+u_{0}^{2}\right)}{\omega_{p e}^{2}+k_{x}^{2} c^{2}}}=\sqrt{\bar{\gamma}_{W I}^{2}+\bar{\gamma}_{C F I}^{2}},
$$

where we recognise the contributions

$$
\bar{\gamma}_{W I} \simeq \omega_{p e} \frac{k_{x} c_{y}}{\sqrt{\omega_{p e}^{2}+k_{x}^{2} c^{2}}}, \quad \bar{\gamma}_{C F I} \simeq \omega_{p e} \frac{k_{x}\left|u_{0}\right|}{\sqrt{\omega_{p e}^{2}+k_{x}^{2} c^{2}}},
$$

which are the well-known growth rates of the WI and of the symmetric cold CFI, when considered separately. Equation (20) is understood if we recover the two limits of eqs. (21) by considering each beam as represented by a bi-Maxwellian distribution function, whose variances in the velocity space, $\sigma_{i}^{2} \equiv k_{B} T_{i} / m$ for $i=x, y$, correspond to $\sigma_{x}^{2}=c_{x}^{2}$ and $\sigma_{y}^{2}=c_{y}^{2}$ as given by eqs. (7). In the limit case $\left|u_{0}\right| \lesssim c_{y}$, for which $\gamma_{W I / C F I} \simeq \bar{\gamma}_{W I}$ (fig. 2(a)), this two-beam configuration approaches a single anisotropic bi-Maxwellian distribution of temperatures $T_{x}<T_{y}$, centered around $v_{x} / c_{y}=0, v_{y} / c_{y} \simeq 0$, that is, the typical initial configuration for the WI with no beams (cf. fig. 3(a)). Conversely, in the limit $\left|u_{0}\right| \gg c_{y}$ for which $\gamma_{W I / C F I} \simeq \bar{\gamma}_{C F I}$ (fig. 2(b)), the neglect of $\sigma_{x}$ and $\sigma_{y}$ leads to the classical cold configuration for the CFI considered by Fried [2], i.e. two Dirac distributions centered around $v_{x}=0$ and $v_{y}=u_{0}$ (fig. $3(\mathrm{~b})$ ).

Pure Weibel instability. - When just one population is considered (label $\alpha=1$, which we will drop from the following expressions), $u_{y}^{(0)}=0$ can be assumed after a Galileian transformation that consistently modifies the value of $\boldsymbol{\Pi}^{(0)}$. For $\boldsymbol{k}=k_{x} \boldsymbol{e}_{x}$ this initial configuration is unstable to a pure Weibel mode with dispersion relation

$\omega^{4}-\omega^{2}\left[\omega_{p e}^{2}+k_{x}^{2}\left(c^{2}+c_{x}^{2}\right)\right]+k_{x}^{2}\left[k_{x}^{2} c^{2} c_{x}^{2}-\omega_{p e}^{2}\left(c_{y}^{2}-c_{x}^{2}\right)\right]=0$.

This is obtained from $D_{y y}=0$, by taking the appropriate limit of eq. (13), which reads

$$
D_{y y}=\frac{\omega^{2}}{c^{2}}-k_{x}^{2}-\frac{\omega_{p e}^{2}}{c^{2}}\left(\frac{\omega^{2}+k_{x}^{2}\left(c_{y}^{2}-c_{x}^{2}\right)}{\omega^{2}-k_{x}^{2} c_{x}^{2}}\right) .
$$
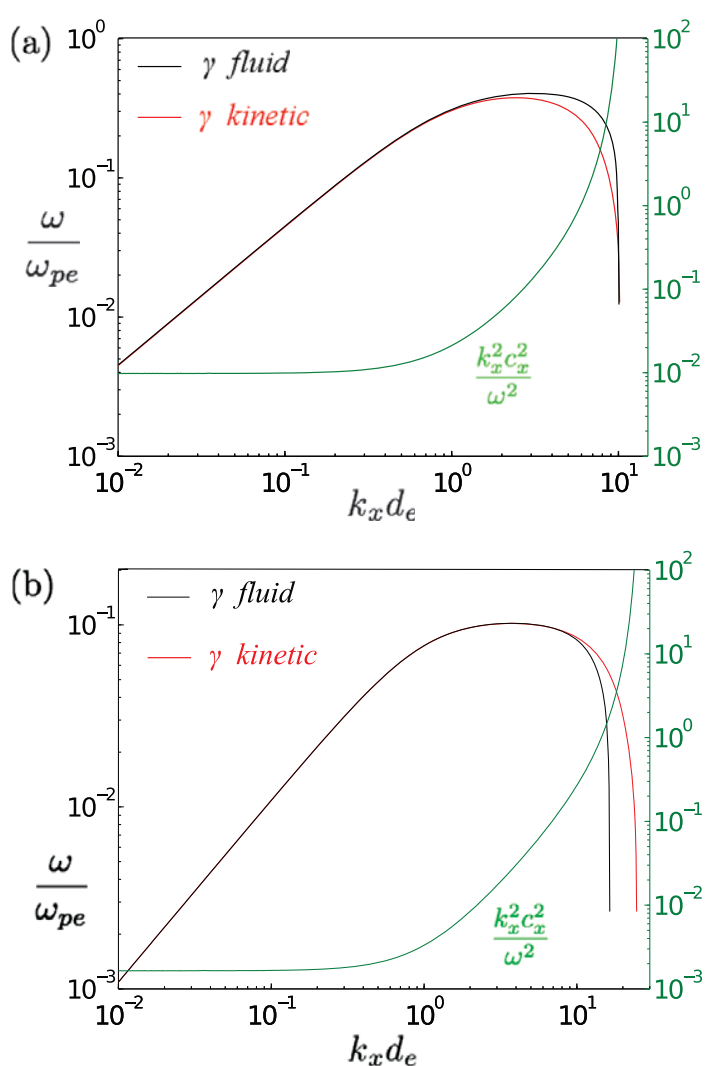

Fig. 2: (Colour online) Fluid (black lines) growth rates of the symmetric WI-CFI mode in the WI-dominated regime (a), for $\left|u_{0}\right|=0.1 c$ and $c_{y}=10 c_{x}=0.442 c$, and in the CFI-dominated regime (b), for $\left|u_{0}\right|=0.1 c$ and $c_{y}=10 c_{x}=0.044 c$ (values $c_{y}^{2} \lesssim 0.2 c^{2}$ have been considered as reasonably non-relativistic). In green the corresponding curve for $\epsilon=k_{x} c_{x} / \omega$ (values on the right axis). The respective kinetic growth rates (red lines) are obtained by integrating the full Vlasov-Maxwell dispersion relation for a double-Maxwellian distribution.

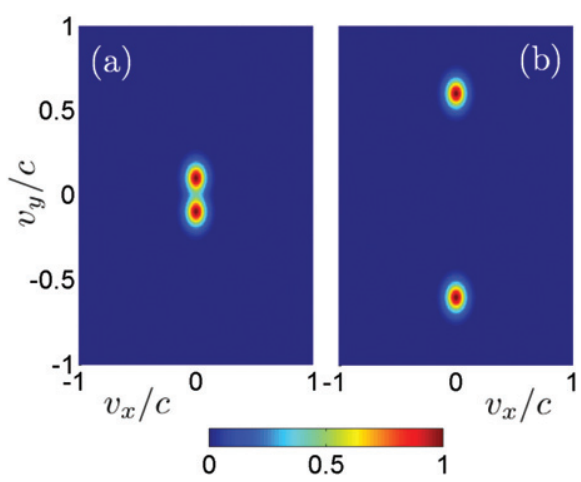

Fig. 3: (Colour online) Contours in velocity space of the electron distribution functions for $\left|u_{0}\right|=\sqrt{3} c_{y}$ (a) and $\left|u_{0}\right| \gg$ $c_{y}(\mathrm{~b})$.

$D_{y y}$ assumes identical form to $D_{z z}$ of eq. (14) previous correspondence $c_{x} \leftrightarrow c_{z} . D_{x x}=0$ gives instead the dispersion relation of Bohm-Gross modes,

$$
\omega^{2}=\omega_{p e}^{2}+3 k_{x}^{2} c_{x}^{2}
$$


After defining $\Delta \equiv\left[\omega_{p e}^{2}+k_{x}^{2}\left(c^{2}-c_{x}^{2}\right)\right]^{2}+4 \omega_{p e}^{2} k_{x}^{2} c_{y}^{2}>0$, the roots of eq. (22) read

$$
\omega_{ \pm}^{2}=\frac{1}{2}\left(\omega_{p e}^{2}+k_{x}^{2}\left(c^{2}+c_{x}^{2}\right) \pm \sqrt{\Delta}\right) .
$$

The WI growth rate,

$$
\gamma_{W I}=\sqrt{\frac{\sqrt{\Delta}}{2}-\frac{\omega_{p e}^{2}+k_{x}^{2}\left(c^{2}+c_{x}^{2}\right)}{2}}
$$

corresponds to $\gamma_{W I}=\left|\omega_{-}\right|$for $\omega_{-}^{2}<0$, condition realised for wave vectors $k_{x} \leq k_{c}^{W I}$, with $k_{c}^{W I}$ given by eq. (19), which solves $\omega\left(k_{c}^{W I}\right)=0$ in eq. (22). The role of temperature anisotropy,

$$
A \equiv \frac{T_{y}}{T_{x}},
$$

appears manifest when we write the instability threshold condition as $A^{2} \geq k_{x}^{2} d_{e}^{2}+1$, which is identical to the one obtained from Vlasov. $A$ also enters in the growth rate $\gamma_{W I}$ through $\Delta=\omega_{p e}^{4}\left(1+k_{x}^{2} d_{e}^{2}\right)^{2}+2 \omega_{p e}^{2} k_{x}^{2} c_{x}^{2}(2 A-1-$ $\left.k_{x}^{2} d_{e}^{2}\right)+k_{x}^{4} c_{x}^{4}$. The solution $\bar{\gamma}_{W I}$ of eqs. (21) is obtained from $\gamma_{W I}$ of eq. (26) in the limit $A \gg 1, c^{2} \gg c_{y}^{2}$.

The kinetic growth rate, obtained by solving numerically Weibel's kinetic dispersion relation [1], $\omega^{2}=$ $c^{2} k_{x}^{2}+\omega_{p e}^{2}\left(1+c_{y}^{2} / c_{x}^{2} W(\xi)\right)$, with $W(\xi) \equiv-(1+\xi Z(\xi))$ and $Z(\xi)$ plasma dispersion function of argument $\xi \equiv$ $\omega /\left(k_{x} \sqrt{2 k_{B} T_{x} / m}\right)$, results smaller than the fluid one, the smaller the anisotropy $A$ is. The cut-off wave vectors coincide in the two models, regardless of whether we retain or not the displacement current, whose neglect makes disappear the electrostatic Bohm-Gross modes but negligbly influence the WI growth rate. We note that Basu [3] already compared the fluid growth rate with Weibel's kinetic result in the strong anisotropy limit. This, however, does not equal the condition $A \gg 1$, since Basu also assumed thermal effects to be small, $\epsilon \equiv k_{x} c_{x} / \omega \ll 1$, whence the strong anisotropy condition follows for $k_{x} d_{e} \ll 1$. Weibel's result for $A \gg 1[1]$ is thus recovered when $\sim \varepsilon^{2}$ terms in eq. (22) are neglected. These are same order of the $k_{x} \tilde{Q}_{x x y}$ contributions, formally considered but then practically neglected in Basu's calculation. When also $\sim \epsilon$ terms are disregarded the cut-off wave vector is obviously lost.

An interesting feature we recognise is the way an initial pressure anisotropy makes it possible the propagation of low-frequency, transverse e.m. waves, otherwise strongly damped in an isotropic plasma if $\omega<\omega_{p e}$. The refractive index derived from $D_{y y}=0$ of eq. (23) reads

$$
N \equiv \frac{k c}{\omega}=\sqrt{1-\frac{\omega_{p e}^{2}}{\omega^{2}}\left(\frac{\omega^{2}-k_{x}^{2}\left(c_{y}^{2}-c_{x}^{2}\right)}{\omega^{2}-k_{x}^{2} c_{x}^{2}}\right)} .
$$

While in the isotropic limit temperature effects just increase the frequency threshold below which e.m. waves are damped (i.e. for $\omega^{2}<\omega_{p e}^{2}+k_{x}^{2} c_{x}^{2}$ rather than for $\left.\omega^{2}<\omega_{p e}^{2}\right)$, the contribution of the $\sim \omega_{p e}^{2} k_{x}^{2}\left(c_{y}^{2}-c_{x}^{2}\right)$ term in eq. (28) allows the propagation of low-frequency waves, too: in the limit $\omega_{p e}^{2} \gg k_{x}^{2} c_{x}^{2}, k_{x}^{2} c_{y}^{2}$, we find $N^{2}>0$ also for $\omega^{2}<k^{2}\left(c_{y}^{2}-c_{x}^{2}\right)$. This feature is related to fact that the pressure tensor dynamics allows the excitation of waves at transverse propagation, which in the limit of warm ions and cold massless electrons have been named "magnetoelastic" [23], and which couple compression effects (first r.h.s. term of eq. (29) below) to e.m. perturbations associated to the quadrupole moment of the distribution function (second r.h.s. term of eq. (29)). This is evidenced by the linearised component " $x y$ " of eq. (3),

$$
\frac{\tilde{\Pi}_{x y}}{m n^{(0)}}=\frac{k_{x} c_{x}^{2}}{\omega} \tilde{u}_{y}-i \frac{e}{m} \frac{c_{y}^{2}-c_{x}^{2}}{\omega} \tilde{B}_{z} .
$$

This enters the $y$-component of the momentum equation

$$
\tilde{u}_{y}=-i \frac{e}{m \omega} \tilde{E}_{y}+\frac{k_{x}}{\omega} \frac{\tilde{\Pi}_{x y}}{m n^{(0)}}
$$

and radically modifies the dispersion relation of e.m. waves because of the $\sim\left(c_{y}^{2}-c_{x}^{2}\right) \tilde{B}_{z} / \omega$ term.

Pure CFI with initially isotropic pressure. - We now consider two opposite beams under the assumption of initial isotropic pressure $\Pi_{i j, \alpha}^{(0)}=P_{\alpha}^{(0)} \delta_{i j}$. This generalises the classical cold fluid model by Fried [2], which is recovered for $P_{\alpha}^{(0)} \rightarrow 0$, and prevents the onset of the classical WI mode. We write $c_{\alpha}^{2}=c_{i, \alpha}^{2}$ for $i=x, y, z$. The rewriting of the coefficients $D_{x x}$ and $D_{x y}$ of eqs. (11), (12) is trivial, while $D_{z z}$ and $D_{y y}$ become

$$
\begin{aligned}
& D_{z z}=\frac{\omega^{2}}{c^{2}}-k_{x}^{2}-\sum_{\alpha} \frac{\omega_{p e, \alpha}^{2}}{c^{2}}\left(\frac{\omega^{2}}{\omega^{2}-k_{x}^{2} c_{\alpha}^{2}}\right), \\
& D_{y y}=D_{z z}-\sum_{\alpha} \frac{\omega_{p e, \alpha}^{2}}{c^{2}}\left(\frac{k_{x}^{2} u_{y, \alpha}^{2}}{\omega^{2}-3 k_{x}^{2} c_{\alpha}^{2}}\right) .
\end{aligned}
$$

The propagative modes related to $D_{z z}=0$ are decoupled from the others and in the cold limit $c_{\alpha} \rightarrow 0$ correspond to usual transverse e.m. waves. The presence of an isotropic pressure modifies their dispersion relation, which, in the symmetric case $c_{1}=c_{2} \equiv c_{s}$, becomes

$$
\omega_{ \pm}^{2}=\frac{1}{2}\left(\omega_{p e}^{2}+k_{x}^{2}\left(c^{2}+c_{s}^{2}\right) \pm \sqrt{\Delta^{*}}\right),
$$

with $\Delta^{*} \equiv \sqrt{\left[\omega_{p e}^{2}+k_{x}^{2}\left(c^{2}+c_{t h}^{2}\right)\right]^{2}-4 k_{x}^{4} c^{2} c_{s}^{2}}$.

The "warm" CFI dispersion relation that solves $D_{x x} D_{y y}-D_{x y}^{2}=0$ consists, in the most general case where $D_{x y} \neq 0$, of a coupling between electrostatic BohmGross-type oscillations (corresponding to $D_{x x}=0$ ) and the purely transverse, e.m., CFI mode which would be obtained for $D_{y y}=0$. The writing of eq. (32) emphasizes the different way, with respect to the pure WI case, in which the presence of the beams modifies the stable e.m. modes given by $D_{z z}=0$. As discussed before for the WI-CFI coupled case, in this non-polytropic regime with initially isotropic pressure, eq. (16) specialises to $\sum_{\alpha} P_{\alpha}^{(0)} u_{y, \alpha}^{(0)}=0$ as a condition for $D_{x y}=0$, i.e., for a purely e.m. CFI. A 
similar coupling $\left(D_{x y} \neq 0\right)$ is well known to be found both in the cold limit [5] and in the warm, kinetic regime [12] as due to relativistic effects when non-symmetric beams are considered.

For warm, perfectly symmetric beams, $D_{y y}=0$ gives a sixth-order polynomial in $\omega$, corresponding to the appropriate limit of eq. (17), rewritable in the form

$$
\begin{aligned}
& \left.\left[\left(\omega^{2}-k_{x}^{2} c^{2}\right)\left(\omega^{2}-k_{x}^{2} c_{s}^{2}\right)-\omega^{2} \omega_{p e}^{2}\right)\right]\left(\omega^{2}-3 k_{x}^{2} c_{s}^{2}\right)= \\
& \omega_{p e}^{2} u_{0}^{2} k_{x}^{2}\left[\omega^{2}-k_{x}^{2} c_{s}^{2}\right] .
\end{aligned}
$$

The first 1.h.s. bracket term in eq. (34), if set to zero, would correspond to $D_{z z}=0$ of eq. (31), which gives the propagative, stable modes of eq. (33), while the second l.h.s. bracket term is the contribution of electron sound waves. The two are coupled by the beam velocity through the r.h.s term, and eq. (34) yelds the cut-off $k_{c}^{C F I}$ (eq. (19)), which differs by a factor $\sqrt{3}$ from the kinetic result, $k_{c, k i n}^{C F I} d_{e}=\left|u_{0}\right| / c_{s}$ (see fig. 2, lower panel).

It is interesting to consider, for comparison, the plasma response when a polytropic closure (eq. (4)) replaces the full pressure dynamics in this isotropic limit: $D_{x x}, D_{x y}$ of eqs. (11), (12) remain unchanged but for the substitution $3 c_{x, \alpha}^{2} \rightarrow \Gamma_{\alpha} c_{\alpha}^{2}$. The same substitution holds for the last term in $D_{y y}$ (eq. (13)). However, suppressing $\tilde{\Pi}_{x y}$ and $\tilde{\Pi}_{x z}$ in the $y$ - and $z$-components of momentum equation (eq. (2)) eliminates the $-k_{x}^{2} c_{x, a}^{2}$ term at the denominators of $D_{y y}$ and $D_{z z}$ (cf., e.g., the isotropic limit of eqs. (29), (30)). The mode described by $D_{z z}=0$ thus becomes a standard transverse e.m. wave in a plasma, $\omega^{2}=k_{x}^{2} c^{2}+\omega_{p e}^{2}$, while for symmetric beams $\left(\Gamma_{\alpha}=\Gamma_{s}\right)$ the $D_{y y}=0$ conditions yelds, instead of eq. (34), the following warm CFI dispersion relation,

$$
\left(\omega^{2}-k_{x}^{2} c^{2}-\omega_{p e}^{2}\right)\left(\omega^{2}-\Gamma_{s} k_{x}^{2} c_{s}^{2}\right)=\omega_{p e}^{2} u_{0}^{2} k_{x}^{2} .
$$

A comparison between a kinetic and a fluid, polytropic description was already performed in ref. [16] for the relativistic CFI. Here we note that the cut-off value $k_{c, p o l}^{C F I}=$ $\sqrt{u_{0}^{2} /\left(\Gamma_{s} c_{s}^{2}\right)-1}$ of eq. (35) further differs from the kinetic result since for $u_{0}^{2}>\Gamma_{s} c_{s}^{2}$ it implies a threshold condition for the warm CFI, $k_{x}^{2} d_{e}^{2}<u_{0}^{2} /\left(\Gamma_{s} c_{s}^{2}\right)+1$. This has no counterpart in the full kinetic treatment and is similar to the $A>1$ condition for WI case (first of eqs. (19)). It can be qualitatively understood along the lines of the "pure WI" limit of the coupled WI-CFI mode, by noting that for $\left|u_{0}\right| \sim c_{s}$ the beam velocity distributions in the phase-space tend to overlap (fig. 3(a)). The intrinsic kinetic difference between CFI and WI seems however lost and related to the lack of information about the quadrupole moment deformation of the distribution function: the polytropic closure forces maintenance of isotropy, whereas eq. (3) implies anisotropization (linear analysis gives $\left.\tilde{\Pi}_{y y}=\tilde{\Pi}_{z z}=\tilde{\Pi}_{x x} / 3\right)$. This "anomaly" of the polytropic limit was not evidenced in literature, before.

In the cold limit $c_{s}=0$, both eqs. (34) and (35) reduce to the standard 4th-order expression of the cold, symmetric CFI dispersion relation [5,6], for which the usual growth rate $\bar{\gamma}_{C F I}$ (eq. (21)) is obtained with no cut-off wave vector.

Discussion and conclusions. - We have shown how the inclusion of the full pressure tensor dynamics allows to retain sufficient information in a fluid model, to make it possible a consistent description of Weibel-type modes, at least in their linear phase. This macroscopic point of view is non-trivial. For example, it allows to identify the condition for a purely transverse e.m. regime of the warm CFI as the requirement of global compression work balance through the surface orthogonal to the perturbation (eq. (16)); differently from a polytropic closure it gives a consistent, though not quantitatively accurate, estimation of the cut-off wave vector for the warm CFI; it allows to easily recognise the coupling bewteen WI and CFI (eqs. (17)-(21)) in a regime in which inclusion of relativistic effects and/or excitation of electrostatic unstable modes in a kinetic description made it less intelligible (cf. previous works on this subject, e.g. refs. $[12,13])$; it makes it possible to understand, in terms of the magnetoelastictype modes (cf. ref. [23]) that can be excited thanks to the "rotation" of the pressure tensor components in a magnetic field (eqs. (28)-(30)), how transverse e.m. waves may propagate in an anisotropic plasma.

While the philosophy behind the kinetic multi-stream model [18] is akin to the reduction procedure met in Hamiltonian theory, when a translation invariance along a direction leads to the conservation of the corresponding component of the canonical momentum, the inclusion of the pressure tensor dynamics in a fluid model allows to extend, even in fully 3D plasmas, the large-scale hydrodynamic approach towards regimes where kinetic effects related to pressure anisotropy are important. This extended fluid model thus provides an alternative to computationally demanding kinetic (PIC or Vlasov) simulations in multi-scale collisionless plasma regimes, where the evolution of large spatial scales and the microscopic dynamics cannot be treated independently.

Clearly, the interest to use this model for numerical simulations depends on how much consistent is the nonlinear description of these kinetic istabilities, subject which will deserve future studies. Discrepancies from Vlasov may be expected close to the saturation stage, when nonlinearly developed small scales approach the cut-off wavelength $k_{c}^{-1}$. These effects appear as more important as "less anisotropic" the initial pressures are, for the case of the WI, and as warmer the beams are, in the case of the CFI. Not surprisingly, it is the thermal spread in the perturbation direction, quantified by $\epsilon=k_{x} c_{x} / \omega$, that matters for the discrepancies between fluid and kinetic estimations of the growth rate next to $k_{c}$ (fig. 2). However, the way these discrepancies, in turn, relate to the neglect of the gradient of the heat flux tensor or rather of some higher-order moment of the distribution function is an issue which deserves further studies and seems to depend on the specific instability considered. For example, following the analy- 
sis of ref. [3] for the pure WI case, after linearisation of the relevant components of eq. (9) while setting $\tilde{\boldsymbol{R}}=0$ it can be verified that at the leading order in $\epsilon^{2}$ heat flux contributions only enter through a $k_{x} \tilde{Q}_{x x y} / \omega=-\epsilon^{2} \tilde{\Pi}_{x y}$ term, which exactly cancels out the $\sim-k_{x}^{2} c_{x}^{2}$ contribution at numerator of the first parenthesis term of eq. (13) by yelding $\tilde{u}_{y}=-i\left[1+\left(k_{x} c_{y} / \omega\right)^{2}\right] e \tilde{E}_{y} /(m \omega)$, instead of the $\tilde{u}_{y}=-i\left[1+k_{x}^{2}\left(c_{y}^{2}-c_{x}^{2}\right) / \omega^{2}\right] e \tilde{E}_{y} /(m \omega)$ result of the $\boldsymbol{\nabla} \cdot \boldsymbol{Q}=0$ case -cf. eq. (30); the $k_{x} \tilde{Q}_{x x x} / \omega=-3 \epsilon^{2} \tilde{\Pi}_{x x}$ correction to the $(x x)$-component of eq. (3) gives instead a negligible $\sim \varepsilon^{4}$ correction in the equation for $\tilde{u}_{x}$ with respect to the $\nabla \cdot Q=0$ case. The "consistent" estimation of both the WI kinetic cut-off wave vector (first of eqs. (19)) and of the refractive index in the presence of thermal anisotropy (eq. (28)) is this way lost. This would suggest the need to retain the contributions of the fourth-order moment $\boldsymbol{R}_{\alpha}$, too, when the heat flux gradient is included, similarly to what happens for the description of kinetic Alfvén eigenmodes [26]. On the other hand, however, the consistent results obtained while neglecting $\boldsymbol{\nabla} \cdot \boldsymbol{Q}$ agree with the successful description of the WI by means of only three initial symmetric beams in the velocity coordinate [18], which implies a strictly null heat flux in the whole linear evolution. More complex can be the analysis of the coupling with electrostatic TSI-modes for arbitrary $\boldsymbol{k} \cdot \boldsymbol{u}^{(0)}$, which would require to reason in terms of $k c_{||} /\left|\omega-\boldsymbol{k} \cdot \boldsymbol{u}_{\alpha}^{(0)}\right|$ rather than in terms of $\epsilon\left(c_{\|}\right.$measuring the thermal spread along $\boldsymbol{k}$ ) and which will be carried out elsewhere. Finally note that Landau-resonances, which could become important for propagation parallel to $\boldsymbol{B}$, e.g., in the whistler or mirror instability, have been also accounted for in Landau-fluid models extended [26-28] to include the approximated pressure tensor response, as it can be derived from eqs. (1)-(3) by power expansion at low frequencies with respect to the cyclotron ones [29].

All these elements draw interest to the use of this fluid approach, which will be next extended to the relativistic regime where the initial phase-space anisotropies are not as easily identifiable in terms of "temperatures": its comparison, not only with nonlinear Vlasov-Maxwell simulations but also with other reduced kinetic models, will allow further insight on the role played by high-order moments of Vlasov equation in the dynamics of plasma microinstabilities.

\section{REFERENCES}

[1] Weibel E., Phys. Rev. Lett., 2 (1959) 83.

[2] Fried B. D., Phys. Fluids, 2 (1959) 337.
[3] Basu B., Phys. Plasmas, 9 (2002) 5131.

[4] Schlickeiser R. and Shukla P. K., Astrophys. J., 599 (2003) L60.

[5] Pegoraro F., Bulanov S. V., Califano F. and Lontano M., Phys. Scr., T63 (1996) 262.

[6] Califano F., Pegoraro F. and Bulanov S. V., Phys. Rev. E, 56 (1997) 963.

[7] Califano F., Prandi R., Pegoraro F. and Bulanov S. V., Phys. Rev. E, 58 (1998) 7837.

[8] Califano F., Pegoraro F., Bulanov S. V. and Mangeney A., Phys. Rev. E, 57 (1998) 7084.

[9] Califano F., Attico N., Pegoraro F., Bertin G. and Bulanov S. V., Phys. Rev. Lett., 86 (2001) 5293.

[10] Califano F., Del Sarto D. and Pegoraro F., Phys. Rev. Lett., 96 (2006) 105008.

[11] Tzoufras M., Ren C., Tsung F. S., Tonge J. W., Mori W. B., Fiore M., Fonseca R. A. and Silva L. O., Phys. Rev. Lett., 96 (2006) 105002.

[12] Bret A., Gremillet L. and Bellido J. C., Phys. Plasmas, 14 (2007) 032103.

[13] Bret A., Gremillet L. and Dieckmann M. E., Phys. Plasmas, 17 (2010) 120501.

[14] Yoon P. H. and Davidson R. C., Phys. Rev. A, 35 (1987) 2718.

[15] Bret A., Phys. Lett. A, 359 (2006) 52.

[16] Bret A. and Deutsch C., Phys. Plasmas 13 (2006) 042106.

[17] Silva O. L., Fonseca R. A., Tonge J. W., Mori W. B. and Dawson J. M., Phys. Plasmas, 9 (2002) 2458.

[18] Inglebert A., Ghizzo A., Reveillé T., Del Sarto D., Bertrand P. and Califano F., EPL, 95 (2011) 45002.

[19] Inglebert A., Ghizzo A., Reveillé T., Bertrand P. and Califano F., Phys. Plasmas 19 (2012) 122109.

[20] Ghizzo A., Phys. Plasmas, 20 (2013) 082109.

[21] Ghizzo A., Phys. Plasmas, 20 (2013) 082110.

[22] Ghizzo A., Phys. Plasmas, 20 (2013) 082111.

[23] Del Sarto D., Pegoraro F. and Tenerani A., arXiv:1509.04938 (2015).

[24] Del Sarto D., Pegoraro F. and Califano F., Phys. Rev. E, 93 (2016) 053203.

[25] Cerri S. S., Pegoraro F., Califano F., Del Sarto D. and Jenko F., Phys. Plasmas, 21 (2014) 112109.

[26] Sulem P. L. and Passot T., J. Plasma Phys., 81 (2015) 325810103

[27] Goswami P., Passot T. and Sulem P. L., Phys. Plasmas, 12 (2005) 102109.

[28] Passot T. and Sulem P. L., Phys. Plasmas, 14 (2007) 082502.

[29] Cerri S. S., Henri P., Califano F., Del Sarto D., Faganello M. and Pegoraro F., Phys. Plasmas, 20 (2013) 112112. 